\title{
Bi-Dimensional Colored Fuzzy Entropy Applied to Melanoma Dermoscopic Images ${ }^{\dagger}$
}

\author{
Andreia Sofia F. Gaudêncio ${ }^{1}$, Mirvana Hilal ${ }^{2}$, Pedro G. Vaz ${ }^{1}$, João M. Cardoso ${ }^{1}$ and Anne \\ Humeau-Heurtier $^{2}$ \\ 1 LIBPhys-UC, Physics Department, University of Coimbra, Coimbra, Portugal \\ 2 LARIS-Laboratoire Angevin de Recherche en Ingénierie des Systèmes, University of Angers, 62 Avenue \\ Notre-Dame du Lac, Angers, France \\ + Presented at the Entropy 2021: The Scientific Tool of the 21st Century, 5-7 May 2021; Available online: \\ https://sciforum.net/conference/Entropy2021/.
}

Published: 5 May 2021

Recently, a bi-dimensional fuzzy entropy measure has been proposed for image texture evaluation. Herein, a new bi-dimensional fuzzy entropy is proposed to process colored images. Our algorithm, FuzEnc2D, in opposition to dos Santos et al. (2018) definition, evaluates each color channel individually with consideration of global and local characteristics. We propose to apply it for the characterization of melanoma's dermoscopic images. In this work, FuzEnc2D is tested by evaluating its sensitivity to change of parameters, rotation sensitivity, ability to determine irregularity through shuffling pixels, and consistency according to different image sizes. For those purposes, white noise and colored Brodatz textures are used. The algorithm is also applied to dermoscopic images of the public PH2 dataset to evaluate its performance in distinguishing common nevi, atypical nevi, and melanoma lesions. The results reveal a relative decrease of, at most, 29.97\% for FuzEnc2D-values when considering different parameters values. On the other hand, the consistency and low rotation sensitivity of the algorithm are revealed by analyzing the same texture with different sizes (maximum relative difference of $4.34 \%$ ) and when comparing the entropy of an image upon rotation (maximum relative difference of $0.36 \%$ ). Besides, after shuffling the pixels of an image, FuzEnc2D-values of shuffled images increases up to 8.9 times of the original values. Moreover, using the red channel's entropy, a common nevi lesion is statistically different from an atypical one ( $p=0.004$ with the Kruskal-Wallis test). Regarding the green channel, a statistical difference $(p=0.034)$ is observed between atypical nevi lesions and melanoma. Also, differentiating a common nevi lesion from lesions diagnosed as melanoma is possible regardless the RGB channels. Finally, the FuzEnc2D algorithm appears as a promising algorithm to analyze, through an entropy-based measure, the texture of colored images.

(C) 2021 by the authors. Licensee MDPI, Basel, Switzerland. This article is an open access article distributed under the terms and conditions of the Creative Commons Attribution (CC BY) license (http://creativecommons.org/licenses/by/4.0/). 